\title{
GMR
}

\section{Identification of disturbed pathways in heart failure based on Gibbs sampling and pathway enrichment analysis}

\author{
P. Chen ${ }^{1}$, L.H. Guo ${ }^{1}$, Y.K. Guo ${ }^{1}$, Z.J. Qu' ${ }^{1}$,Y. Gao ${ }^{2}$ and H. Qiu ${ }^{2}$ \\ ${ }^{1}$ Department of Cardiology, \\ Jinan Municipal Hospital of Traditional Chinese Medicine, \\ Jinan, Shandong Province, China \\ ${ }^{2}$ Department of Internal Medicine, \\ Jinan Municipal Hospital of Traditional Chinese Medicine, \\ Jinan, Shandong Province, China \\ Corresponding author: P. Chen \\ E-mail: aichenping2015@sina.com
}

Genet. Mol. Res. 15 (2): gmr.15027956

Received October 29, 2015

Accepted January 15, 2016

Published April 27, 2016

DOI http://dx.doi.org/10.4238/gmr.15027956

\begin{abstract}
We identified disturbed pathways in heart failure (HF) based on Gibbs sampling combined with pathway enrichment analysis. A total of 396 Markov chains (MCs) (gene count $>5$ ) were obtained. After Gibbs sampling, six differentially expressed molecular functions (DEMFs) (possibility $\geq 0.8$ ) were obtained. As statistical analysis was performed on the number of individual differentially expressed genes (DEGs), we found that there were 137 DEGs with frequency of occurrence $\geq 2$ in the DEMFs. Pathway enrichment analysis showed that these 137 DEGs were enriched in eight significant pathways under the condition of $\mathrm{P}<0.001$. The five most significant pathways were: the calcium signaling pathway $(\mathrm{P}=9.08 \mathrm{E}-19)$, arrhythmogenic right ventricular cardiomyopathy $(\mathrm{P}=5.66 \mathrm{E}-13)$, cardiac muscle contraction $(\mathrm{P}=8.04 \mathrm{E}-13)$, hypertrophic cardiomyopathy $(\mathrm{P}=2.55 \mathrm{E}-12)$, and dilated cardiomyopathy $(\mathrm{P}=7.30 \mathrm{E}-12)$. In conclusion, this novel
\end{abstract}


method for identifying significant pathways in HF based on Gibbs sampling combined with pathway enrichment analysis was suitable. We predict that several altered pathways (such as the calcium signaling pathway and dilated cardiomyopathy) may play important roles in HF and are potentially novel predictive and prognostic markers for HF.

Key words: Heart failure; Gibbs sampling; Bayesian inference; Markov chain Monte Carlo; Molecular functions

\section{INTRODUCTION}

Heart failure (HF) is one of the leading causes of death and hospitalization worldwide (Setoguchi et al., 2007). The clinical syndrome of HF is the result of heterogeneous myocardial or vascular diseases, and is defined as the inability to maintain blood circulation throughout the body (McMurray et al., 2014). Although survival after HF diagnosis has improved, HF still has a poor prognosis and $30-40 \%$ of people diagnosed with HF die within one year (Taylor et al., 2014).

Several biomarkers are associated with the development of HF: ST2 (Weinberg et al., 2003), growth differentiation factor 15 (GDF-15) (Anand et al., 2010), TNF- $\alpha$ (Moe et al., 2004), pentraxin 3 (PTX3) (Norata et al., 2009) and AAV1/SERCA2a (Zsebo et al., 2014) have gained interest as biomarkers in HF. The gene expression profiling that reveals potential prognostic biomarkers associated with the progression of HF has been identified (Maciejak et al., 2015). However, there is still a lack of understanding about the mechanism underlying HF. That knowledge would facilitate the customization of novel treatment strategies for the disorder and uncover potential therapeutic targets to slow HF progression to improve the quality of life and reduce mortality.

Genes are tightly regulated to execute the proper biological functions of a cell in order to respond to internal or external perturbations (Kostka and Spang, 2004). Therefore, their expression variations during the disease deterioration process are causally associated with phenotype changes. In general, differentially expressed genes (DEGs) can provide clues about the pathogenesis of destructive pathogen (Liu et al., 2013). However, DEGs alone may lead to false positives because some genes show significant expression differences due to certain stimuli, but are not related to the pathogenic pathway (MacFarlane and Singh, 2006). Therefore, whole-level analysis is necessary and pathway analysis has become the first choice for gaining insight into the underlying biology of DEGs and proteins (Goeman and Bühlmann, 2007). Gibbs sampling, a Markov chain Monte Carlo (MCMC) algorithm for obtaining a sequence of observations that are approximated from a specified multivariate probability distribution (Walsh, 2004), can be used to identify differentially expressed molecular functions (DEMFs).

Therefore, in this paper, Gibbs sampling combined with pathway analysis was applied to identify disturbed pathways in HF. To achieve this, firstly the gene expression profile of HF was acquired and preprocessed. Secondly, the genes were transformed to Markov chains (MCs) to conduct Gibbs sampling. Gibbs sampling was then performed to identify the DEMFs. Finally, pathway enrichment analysis for the genes associated with the DEMFs was carried out based on Database for Annotation, Visualization and Integrated Discovery (DAVID), so as to better understand the underlying mechanism of HF. 


\section{MATERIAL AND METHODS}

\section{Data acquisition and preprocessing}

The preprocessed gene expression profile of HF was downloaded from the ArrayExpress database (http://www.ebi.ac.uk/arrayexpress/), with accession number E-GEOD-59867 (Maciejak et al., 2015). It was found on the A-AFFY-141-Affymetrix GeneChip Human Gene 1.0 ST Array [HuGene-1_0-st-v1] Platform, and comprised 80 samples (46 normal controls and $34 \mathrm{HF}$ samples). The microarray data and annotation files were downloaded for further analysis. The gene expression profile at the probe level was converted to the gene symbol level, and the duplicated symbols were wiped off; 18977 gene symbols were obtained for further analysis.

\section{Identifying molecular functions (MFs)}

To implement the Gibbs sampling, the data set must be transformed to a data set with functional class expression measurements (Quiroz-Zárate et al., 2015). The Gene Ontology (GO) project (http://www.geneontology.org/), which is freely available for community use in the annotation of genes, gene products, and gene sequences, can provide structured, controlled vocabularies and classifications that cover several domains of molecular and cellular biology (Gene Ontology Consortium, 2004). Moreover, the GO MF terms represent activities rather than entities (molecules or complexes) that perform actions, and do not specify where, when, or in what context the action takes place (Ashburner et al., 2000). Therefore, the gene function of the preprocessed data was classed via the GO project, and all of the genes were distributed to the MFs. Only MFs from GOs with at least five genes were considered for further analysis. All the MFs were then converted into MCs using the MCMCDataSet function of the BAGS package.

\section{Posterior inference}

After having transformed all the MFs into MCs, their posterior inferences were defined to identify probability distributions of MFs for HF (Moradkhani et al., 2012). The Bayesian framework, which is implemented using the MCMC method, not only provides the marginal and joint posterior distributions of all the unknown parameters, but also presents a convenient means of incorporating parameter uncertainty into predictive inferences (Neuwald, 2014). Given the observations, $y=\left(y_{1}, \ldots, y_{n}\right)$, the posterior distribution of $\beta, \pi(b \mid y)$ is given by:

$$
\pi(\beta \mid y) \propto L(y \mid \beta) p(\beta)
$$

Where $p(\beta)$ is the prior distribution of $\beta$ and $\mathrm{L}(\mathrm{y} \mid \beta)$ is the likelihood function written as:

$$
L(y \mid \beta)=p^{n}(1-p)^{n} \exp \left\{-\sum_{i} \rho_{p}\left(y_{i}-x_{i}^{\prime} \beta\right)\right\}
$$




\section{Gibbs sampling}

To implement Gibbs sampling, we needed to define the empty objects in which the posterior samples of the parameters of interest were to be kept. In the present research, Gibbs 2 function of the BAGS package provided the MCs for the parameters of interest that would form their posterior distribution, and this function was used to obtain the differentially expressed gene sets among the normal control and disease groups (Quiroz-Zárate et al., 2013). First of all, an empty Gibbs sampling set comprising a $k$-dimensional $(k=10,000)$ random vector was defined. Secondly, $N$ samples ( $N=$ MFs identified above) were put into the empty Gibbs sampling set. Thirdly, $k$ cycles of randomly fixed $k-1$ elements of the vector, were extracted from the remaining element to gain a new sample set. Finally, $N$ cycles of the second step were applied to obtain new MCs. The possibility of an MF appearing $k$ times, or the posterior value of an MF was calculated according to the following formula:

Possibility $=$ average P value of the MFs (from 2000 to 10,000) $\times 250 /(10,000-2000+1)$

\section{Identifying DEMFs}

After obtaining the posterior values of the MFs, a possibility value of 0.8 was selected as the cut-off criteria, and the MFs with possibility values $\geq 0.8$ were regarded as DEMFs. To further investigate the properties of the DEMFs, the gene compositions of the DEMFs were studied, and genes involved in the DEMFs were considered DEGs. Statistical analysis was then performed on the number of individual DEGs. The genes that appeared $\geq 2$ times were considered critical DEGs and were chosen for in-depth analysis.

\section{Pathway enrichment analysis of the DEGs}

The Kyoto Encyclopedia of Genes and Genomes (KEGG) represents an effort to link genomic information with higher order functional information by computerizing current knowledge of cellular processes and standardizing gene annotations (Kanehisa and Goto, 2000). In this study, the KEGG database was used to investigate the enrichment analysis of DEGs involved in the occurrence and development of HF. The DAVID database (Huang et al., 2007) was used to perform the KEGG pathway enrichment analysis. Pathways with P values $<0.001$ and gene counts $>5$ were selected according to the expression analysis systematic explorer (EASE) test implemented in DAVID (Wang and Simon, 2011).

\section{RESULTS}

\section{Identifying differentially expressed MFs and DEGs}

In this paper, Gibbs sampling was used to analyze the expression profile of HF. The BAGS package was applied to analyze DEMF identification. By setting the threshold value of the gene count to $>5$, we obtained $396 \mathrm{MFs}$ in total. After obtaining the posterior value of the MFs by Gibbs sampling, and choosing a threshold value of possibility of 0.8 , as shown in Figure 1, we obtained six DEMFs as follows: inositol or phosphatidylinositol phosphatase activity (possibility $=0.96$ ), lipoprotein binding (possibility $=0.93$ ), small conjugating protein binding (alfa.pi $=0.85$ ), gated channel activity (possibility $=0.85$ ), receptor activity 
(possibility $=0.82$ ), and ion channel activity (possibility $=0.82$ ). Statistical analysis on the number of individual DEGs revealed that 137 DEGs had a frequency of occurrence $\geq 2$ in the DEMFs, and those 137 DEGs were extracted for further analysis.

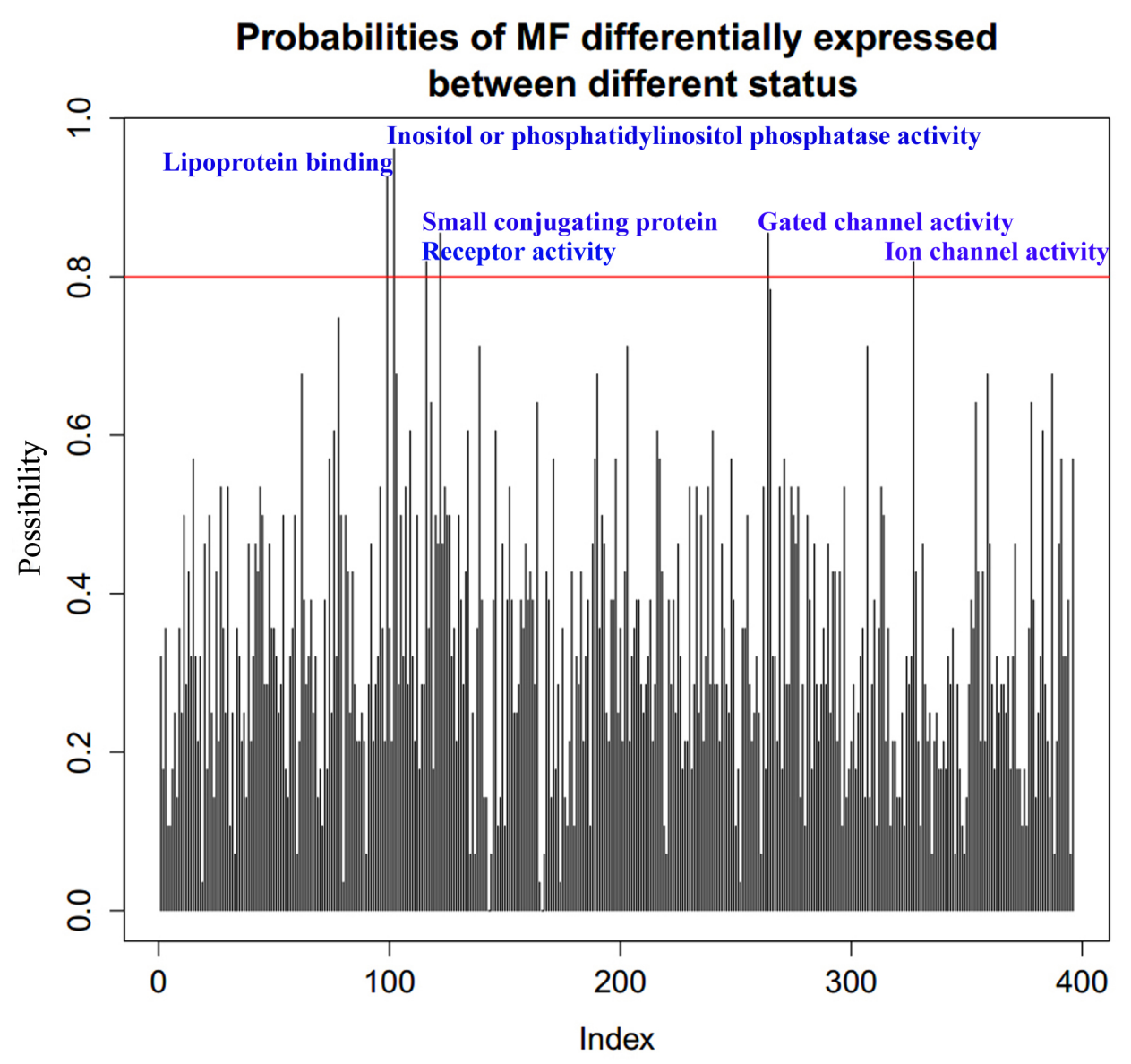

Figure 1. Probabilities for 396 molecular functions (MFs). The horizontal ordinate represents the MFs, and the vertical coordinate represents the posterior value of the MFs.

\section{Pathway enrichment analysis}

To gain further insights into the function of the DEGs, DAVID was applied to identify the significantly disturbed KEGG pathways. By setting the threshold value of the $\mathrm{P}$ value to $<0.001$ and the gene count to $>5$, the results showed that 137 DEGs were enriched in eight significant pathways (Table 1). The most significant five pathways were the calcium signaling pathway $(\mathrm{P}=9.08 \mathrm{E}-19)$, arrhythmogenic right ventricular cardiomyopathy (ARVC) $(\mathrm{P}=$ 5.66E-13), cardiac muscle contraction $(\mathrm{P}=8.04 \mathrm{E}-13)$, hypertrophic cardiomyopathy (HCM) $(\mathrm{P}=2.55 \mathrm{E}-12)$, and dilated cardiomyopathy $(\mathrm{P}=7.30 \mathrm{E}-12)$. 
Table 1. Kyoto Encyclopedia of Genes and Genomes (KEGG) pathway enrichment analysis of the differentially expressed genes (DEGs) $(\mathrm{P}<0.001)$.

\begin{tabular}{l|l|c|c}
\hline ID & Term & Count & P value \\
\hline hsa04020 & Calcium signaling pathway & 23 & $9.08 \mathrm{E}-19$ \\
\hline hsa05412 & Arrhythmogenic right ventricular cardiomyopathy (ARVC) & 14 & $5.66 \mathrm{E}-13$ \\
\hline hsa04260 & Cardiac muscle contraction & 14 & $8.04 \mathrm{E}-13$ \\
\hline hsa05410 & Hypertrophic cardiomyopathy (HCM) & 14 & $2.55 \mathrm{E}-12$ \\
\hline hsa05414 & Dilated cardiomyopathy & 14 & $7.30 \mathrm{E}-12$ \\
\hline hsa04010 & MAPK signaling pathway & 18 & $1.19 \mathrm{E}-09$ \\
\hline hsa04080 & Neuroactive ligand-receptor interaction & 16 & $4.37 \mathrm{E}-08$ \\
\hline hsa04930 & Type II diabetes mellitus & 6 & $1.34 \mathrm{E}-04$ \\
\hline
\end{tabular}

\section{DISCUSSION}

HF is a cardiovascular disease characterized by abnormalities in ventricular function, specifically left ventricular ejection caused by injuries to the heart structure (Duan et al., 2015). Meta-analysis has been used to identify DEGs and the biological processes associated with changes in gene expression in disease (Ning et al., 2015). The traditional methods for identifying DEGs are mainly based on linear models of a microarray data package (Gentleman et al., 2004) or significance analysis of microarrays (Tusher et al., 2001). Traditionally, the analysis of HF has been conducted by identifying the DEGs in the myocardium of HF patients using human whole genomic oligonucleotide microarray-assisted pathway analysis (Wu et al., 2009). In the present paper, Gibbs sampling, an MCMC algorithm that is based on Bayesian theory, was applied to identify DEMFs, to determine disrupted genes and pathways in HF. This was a novel application of the method for studying HF.

By applying Gibbs sampling to research the gene expression profile of HF, we successfully identified six DEMFs. Furthermore, statistical analysis was performed on the number of individual DEGs, and 137 key DEGs were identified. Moreover, by performing KEGG pathway enrichment analysis on the 137 key DEGs, we obtained eight significant pathways $(\mathrm{P}<0.001)$, and the most significant was the calcium signaling pathway $(\mathrm{P}=9.08 \mathrm{E}-$ 19). To better understand the relationship between the occurrence and development of HF and the most significant pathway, we have included an in-depth discussion, as follows.

Ionized calcium $\left(\mathrm{Ca}^{2+}\right)$ is the most common signal transduction element in cells ranging from bacteria to specialized neurons. Unlike many other second-messenger molecules, $\mathrm{Ca}^{2+}$ is required for life, yet prolonged high intracellular $\mathrm{Ca}^{2+}$ levels lead to cell death (Clapham, 2007). Furthermore, $\mathrm{Ca}^{2+}$, via the activation of various kinases and phosphatases, acts on $\mathrm{Ca}^{2+}$ dependent transcription pathways (Lompré et al., 2010). $\mathrm{Ca}^{2+}$-dependent signaling is highly regulated in cardiomyocytes and determines the force of cardiac muscle contraction. There is clear evidence that alterations in sarcoplasmic reticulum (SR) $\mathrm{Ca}^{2+}$ cycling are involved in the impaired contractile performance of the failing heart (Hasenfuss, 1998), and the role of a pathological diastolic $\mathrm{SR} \mathrm{Ca}{ }^{2+}$ leak via calstabin2-depleted leaky ryanodine receptor 2 (RyR2) has been recognized as an important contributor to altered $\mathrm{Ca}^{2+}$ handling in $\mathrm{HF}$ (Wehrens et al., 2005). Currently, two new therapeutic strategies for HF are being tested in clinical trials: (a) fixing the leak in RyR2 channels with a novel class of $\mathrm{Ca}^{2+}$-release channel stabilizers called Rycals; and (b) increasing the expression of sarcoplasmic/endoplasmic reticulum $\mathrm{Ca}^{2+}$ ATPase to improve SR $\mathrm{Ca}^{2+}$ reuptake with viral-mediated gene therapy (Marks, 2013). In the present study, by performing analysis on the HF gene expression profile using Gibbs sampling 
combined with KEGG pathway enrichment analysis, we found that calcium signaling ( $\mathrm{P}=$ 9.08E-19) was the most significant pathway, which indirectly illustrated the applicability of the method.

Arrhythmogenic right ventricular cardiomyopathy (ARVC) $(\mathrm{P}=5.66 \mathrm{E}-13)$, cardiac muscle contraction $(\mathrm{P}=8.04 \mathrm{E}-13)$, hypertrophic cardiomyopathy $(\mathrm{HCM})(\mathrm{P}=2.55 \mathrm{E}-12)$, and dilated cardiomyopathy $(\mathrm{P}=7.30 \mathrm{E}-12)$ are recognized as significant pathways in the occurrence and development of $\mathrm{HF}$, and further discussions were conducted on the relationship between HF and these pathways. ARVC is a structural cardiomyopathy of genetic origin, and is characterized by structural and functional abnormalities of the right ventricle, ranging from regional wall motion abnormalities and aneurysms to global dilation and dysfunction, with or without left ventricular involvement (Corrado et al., 2011). Idiopathic dilated cardiomyopathy is a form of HF defined by the presence of left ventricular dilatation and left ventricular systolic dysfunction in the absence of an obvious etiology, such as coronary artery disease, hypertension, valvular disease, or congenital defect (Jefferies and Towbin, 2010). Dilated cardiomyopathy is a major cause of systolic HF and the main indication for heart transplantation (Villard et al., 2011). Gadolinium cardiovascular magnetic resonance can exclude the presence of left ventricular dysfunction related to coronary artery disease in heart failure (McCrohon et al., 2003).

In conclusion, this novel method for identifying significant pathways in HF based on Gibbs sampling combined with KEGG pathway enrichment analysis was suitable. We predict that several altered pathways (such as the calcium signaling pathway and dilated cardiomyopathy) may play important roles in HF and are potentially novel predictive and prognostic markers for HF. However, there were some limitations to our study. First, the preprocessed gene expression profile of HF was downloaded from an existing database rather than obtained by ourselves. Secondly, further experimental verification should be conducted to verify our results.

\section{Conflicts of interest}

The authors declare no conflict of interest.

\section{ACKNOWLEDGMENTS}

Research supported by the Department of Cardiology and Internal Medicine, Jinan Municipal Hospital of Traditional Chinese Medicine. We thank all members of the research group. We are also grateful to the Ji'nan Evidence Based Medicine Science-Technology Center for technical assistance and critical reading of the manuscript.

\section{REFERENCES}

Anand IS, Kempf T, Rector TS, Tapken H, et al. (2010). Serial measurement of growth-differentiation factor-15 in heart failure: relation to disease severity and prognosis in the Valsartan Heart Failure Trial. Circulation 122: 1387-1395. http://dx.doi.org/10.1161/CIRCULATIONAHA.109.928846

Ashburner M, Ball CA, Blake JA, Botstein D, et al.; The Gene Ontology Consortium (2000). Gene ontology: tool for the unification of biology. Nat. Genet. 25: 25-29. http://dx.doi.org/10.1038/75556

Clapham DE (2007). Calcium signaling. Cell 131: 1047-1058. http://dx.doi.org/10.1016/j.cell.2007.11.028

Corrado D, Basso C, Pilichou K and Thiene G (2011). Molecular biology and clinical management of arrhythmogenic right ventricular cardiomyopathy/dysplasia. Heart 97: 530-539. http://dx.doi.org/10.1136/hrt.2010.193276 
Duan HY, Liu DM, Qian P, Wang SL, et al. (2015). Effect of atorvastatin on plasma NT-proBNP and inflammatory cytokine expression in patients with heart failure. Genet. Mol. Res. 14: 15739-15748. http://dx.doi.org/10.4238/2015. December.1.25

Gene Ontology Consortium (2004). The Gene Ontology (GO) database and informatics resource. Nucleic Acids Res. 32: D258-D261. http://dx.doi.org/10.1093/nar/gkh036

Gentleman RC, Carey VJ, Bates DM, Bolstad B, et al. (2004). Bioconductor: open software development for computational biology and bioinformatics. Genome Biol. 5: R80. http://dx.doi.org/10.1186/gb-2004-5-10-r80

Goeman JJ and Bühlmann P (2007). Analyzing gene expression data in terms of gene sets: methodological issues. Bioinformatics 23: 980-987. http://dx.doi.org/10.1093/bioinformatics/btm051

Hasenfuss G (1998). Alterations of calcium-regulatory proteins in heart failure. Cardiovasc. Res. 37: 279-289. http:// dx.doi.org/10.1016/S0008-6363(97)00277-0

Huang DW, Sherman BT, Tan Q, Collins JR, et al. (2007). The DAVID Gene Functional Classification Tool: a novel biological module-centric algorithm to functionally analyze large gene lists. Genome Biol. 8: R183. http://dx.doi. org/10.1186/gb-2007-8-9-r183

Jefferies JL and Towbin JA (2010). Dilated cardiomyopathy. Lancet 375: 752-762. http://dx.doi.org/10.1016/S0140$\underline{6736(09) 62023-7}$

Kanehisa M and Goto S (2000). KEGG: kyoto encyclopedia of genes and genomes. Nucleic Acids Res. 28: 27-30. http:// dx.doi.org/10.1093/nar/28.1.27

Kostka D and Spang R (2004). Finding disease specific alterations in the co-expression of genes. Bioinformatics 20 (Suppl 1): i194-i199. http://dx.doi.org/10.1093/bioinformatics/bth909

Liu Y, Chen SH, Jin X and Li YM (2013). Analysis of differentially expressed genes and microRNAs in alcoholic liver disease. Int. J. Mol. Med. 31: 547-554.

Lompré AM, Hajjar RJ, Harding SE, Kranias EG, et al. (2010). Ca2+ cycling and new therapeutic approaches for heart failure. Circulation 121: 822-830. http://dx.doi.org/10.1161/CIRCULATIONAHA.109.890954

MacFarlane RC and Singh U (2006). Identification of differentially expressed genes in virulent and nonvirulent Entamoeba species: potential implications for amebic pathogenesis. Infect. Immun. 74: 340-351. http://dx.doi.org/10.1128/ IAI.74.1.340-351.2006

Maciejak A, Kiliszek M, Michalak M, Tulacz D, et al. (2015). Gene expression profiling reveals potential prognostic biomarkers associated with the progression of heart failure. Genome Med. 7: 26. http://dx.doi.org/10.1186/s13073$\underline{015-0149-\mathrm{Z}}$

Marks AR (2013). Calcium cycling proteins and heart failure: mechanisms and therapeutics. J. Clin. Invest. 123: 46-52. http://dx.doi.org/10.1172/JCI62834

McCrohon JA, Moon JC, Prasad SK, McKenna WJ, et al. (2003). Differentiation of heart failure related to dilated cardiomyopathy and coronary artery disease using gadolinium-enhanced cardiovascular magnetic resonance. Circulation 108: 54-59. http://dx.doi.org/10.1161/01.CIR.0000078641.19365.4C

McMurray JJ, Packer M, Desai AS, Gong J, et al.; PARADIGM-HF Investigators and Committees (2014). Angiotensinneprilysin inhibition versus enalapril in heart failure. N. Engl. J. Med. 371: 993-1004. http://dx.doi.org/10.1056/ NEJMoa1409077

Moe GW, Marin-Garcia J, Konig A, Goldenthal M, et al. (2004). In vivo TNF-alpha inhibition ameliorates cardiac mitochondrial dysfunction, oxidative stress, and apoptosis in experimental heart failure. Am. J. Physiol. Heart Circ. Physiol. 287: H1813-H1820. http://dx.doi.org/10.1152/ajpheart.00036.2004

Moradkhani H, DeChant CM and Sorooshian S (2012). Evolution of ensemble data assimilation for uncertainty quantification using the particle filter-Markov chain Monte Carlo method. Water Resour. Res. 48: W12520. http:// dx.doi.org/10.1029/2012WR012144

Neuwald AF (2014). A Bayesian sampler for optimization of protein domain hierarchies. J. Comput. Biol. 21: 269-286. http://dx.doi.org/10.1089/cmb.2013.0099

Ning LF, Yu YQ, GuoJi ET, Kou CG, et al. (2015). Meta-analysis of differentially expressed genes in autism based on gene expression data. Genet. Mol. Res. 14: 2146-2155. http://dx.doi.org/10.4238/2015.March.27.1

Norata GD, Marchesi P, Pulakazhi Venu VK, Pasqualini F, et al. (2009). Deficiency of the long pentraxin PTX3 promotes vascular inflammation and atherosclerosis. Circulation 120: 699-708. http://dx.doi.org/10.1161/ CIRCULATIONAHA.108.806547

Quiroz-Zárate A, Haibe-Kains B and Quackenbush J (2015). BAGS: A Bayesian Approach for Geneset Selection. Available at [https://www.bioconductor.org/packages/3.3/bioc/vignettes/BAGS/inst/doc/BAGS.pdf]. Accessed January 12, 2016.

Quiroz-Zárate A, Quiroz-Zárate MA, Gibbs CDRGR and GrpMean RGRGR (2013). Package 'BAGS'. 
Setoguchi S, Stevenson LW and Schneeweiss S (2007). Repeated hospitalizations predict mortality in the community population with heart failure. Am. Heart J. 154: 260-266. http://dx.doi.org/10.1016/j.ahj.2007.01.041

Taylor RS, Sagar VA, Davies EJ, Briscoe S, et al. (2014). Exercise-based rehabilitation for heart failure. Cochrane Database Syst. Rev. 4: CD003331.

Tusher VG, Tibshirani R and Chu G (2001). Significance analysis of microarrays applied to the ionizing radiation response. Proc. Natl. Acad. Sci. USA 98: 5116-5121. http://dx.doi.org/10.1073/pnas.091062498

Villard E, Perret C, Gary F, Proust C, et al.; Cardiogenics Consortium (2011). A genome-wide association study identifies two loci associated with heart failure due to dilated cardiomyopathy. Eur. Heart J. 32: 1065-1076. http://dx.doi. org/10.1093/eurheartj/ehr105

Walsh B (2004). Markov chain Monte Carlo and Gibbs sampling. Available at [http://citeseerx.ist.psu.edu/viewdoc/ summary?doi=10.1.1.131.4064].

Wang X and Simon R (2011). Microarray-based cancer prediction using single genes. BMC Bioinformatics 12: 391. http:// dx.doi.org/10.1186/1471-2105-12-391

Wehrens XH, Lehnart SE, Reiken S, van der Nagel R, et al. (2005). Enhancing calstabin binding to ryanodine receptors improves cardiac and skeletal muscle function in heart failure. Proc. Natl. Acad. Sci. USA 102: 9607-9612. http:// dx.doi.org/10.1073/pnas.0500353102

Weinberg EO, Shimpo M, Hurwitz S, Tominaga S, et al. (2003). Identification of serum soluble ST2 receptor as a novel heart failure biomarker. Circulation 107: 721-726. http://dx.doi.org/10.1161/01.CIR.0000047274.66749.FE

Wu XX, Wan T, Wu HJ, Zhi G, et al. (2009). [Identification of differentially expressed genes in myocardium of patients with heart failure by human whole genomic oligonucleotide microarray-assisted pathways analysis]. Zhonghua. Xin Xue Guan Bing Za Zhi 37: 120-125.

Zsebo K, Yaroshinsky A, Rudy JJ, Wagner K, et al. (2014). Long-term effects of AAV1/SERCA2a gene transfer in patients with severe heart failure: analysis of recurrent cardiovascular events and mortality. Circ. Res. 114: 101-108. http://dx.doi.org/10.1161/CIRCRESAHA.113.302421 